\title{
Electrode displacement after intracerebral hematoma as a complication of a deep brain stimulation procedure
}

\author{
Domenico Servello' \\ Marco Sassi' \\ Stefano Bastianello ${ }^{2}$ \\ Guy Umberto Poloni \\ Francesca Mancini ${ }^{3}$ \\ Claudio Pacchetti ${ }^{3}$ \\ 'Functional Neurosurgery Unit, \\ Istituto di Ricovero e Cura a \\ Carattere Scientifico Galeazzi, \\ Milan, Italy; ${ }^{2}$ Neuroradiology Unit; \\ ${ }^{3}$ Parkinson Disease and Movement \\ Disorder Unit, Istituto di Ricovero e \\ Cura a Carattere Scientifico Mondino, \\ Institute of Neurology, Pavia, Italy
}

Objectives: Deep brain stimulation (DBS) is nowadays considered a safe and effective procedure for various movement disorders in which conservative treatments have failed to show significant therapeutic results. One of the most common complications of definitive electrode positioning is intraparenchymal hemorrhage.

Materials and methods: Authors report the case of a 55-year-old female patient treated for Parkinson's disease in which intraparenchymal hemorrhage developed after DBS procedure, leading to significant (about $8 \mathrm{~mm}$ at the neuroradiological controls) displacement of an otherwise correctly positioned DBS electrode.

Results: After conservative management, the hematoma spontaneously resolved. Late neuroradiological controls documented correct, symmetrically positioned electrodes, comparable to the immediate postoperative controls.

Conclusions: Six months follow-up endpoint results of the DBS treatment were considered satisfying by an independent neurologist, with modest residual neurological deficits, demonstrating that re-positioning of the electrode was unnecessary in this rare complication.

Keywords: deep brain stimulation, electrodes, outcomes, implant, case report

\section{Introduction}

Deep brain stimulation (DBS) procedure is considered a safe and effective therapeutic opportunity for patients affected with Parkinson's disease (PD), such that a well experimented algorithm has been developed to determine surgical indication (the CAPSIT examination). Nevertheless, DBS must be considered a surgical procedure, with possible surgically-related complications which might lead to a post-operative disability and mortality. ${ }^{1}$ The most important adverse event related to the DBS procedure is undoubtedly intracranial hemorrhage. Authors present the case of a female patient who was treated for PD with DBS and developed post-surgical intraparenchymal hematoma with a displacement of the omolateral definitive electrode (neuroradiologically well documented with computed tomography $[\mathrm{CT}]$ and X-rays). There is a lack of guidelines concerning neurosurgical management of such a rare complication, and the indication to perform re-positioning of the electrode is thus questionable.

\section{Case report}

A 55-year-old woman (AMC) was diagnosed with idiopathic PD at the age of 49 at the Parkinson's disease neurological ward of our Neurosurgical Division by a dedicated neurologist (CP). Since the age of 52, unwanted dopamine agonists effects occurred, and the disease progressively worsened with motor complications. In January 2006, after proper evaluation (CAPSIT protocol), AMC underwent functional neurosurgery for the implantation of electrodes for bilateral subthalamic nucleus stimulation (STN-DBS). 
At the pre STN-DBS evaluation, the Unified Parkinson's Disease Rating Scale ${ }^{2}$ part III score in ON was 18 and 34 in OFF phase. Involuntary movements were present, with a score of 1 at the Goetz scale. ${ }^{3}$ Mean daily time spent in OFF phase lasted three hours. The Mini Mental State Examination ${ }^{4}$ score was 30 (range 0-30). Clinical features were characterized by asymmetric plastic hypertonia and hypokinesia and a severe trunk dystonia that worsened during standing and during OFF phase. The antiparkinsonian therapy consisted of levodopa/ carbidopa 250/25 mg four times a day, entacapone $200 \mathrm{mg}$ four times a day, and $4 \mathrm{mg}$ of cabergoline twice a day.

Pre-operative study included $3 \mathrm{~mm}$-sliced, gadoliniumenhanced T1 axial and sagittal magnetic resonance imaging (MRI) and T2 coronal MRI scans of the brain, and a $3 \mathrm{~mm}$-sliced CT scan performed with the stereotactic frame. The imaging was fused with a neuronavigation device (Stealthstation; Medtronic Sofamor Danek, Memphis, TN, USA) together with the Schaltenbrand-Wahren Atlas in a well-experimented system, ${ }^{5}$ in order to obtain the stereotactic trajectory with the standard subthalamus coordinates of $12 \mathrm{~mm}$ lateral to, $4 \mathrm{~mm}$ posterior at the midcommissural point, and $4 \mathrm{~mm}$ under the intercommissual plane.

DBS procedure was performed under local anesthesia, with the aid of multi (5)-track microrecording and subsequent

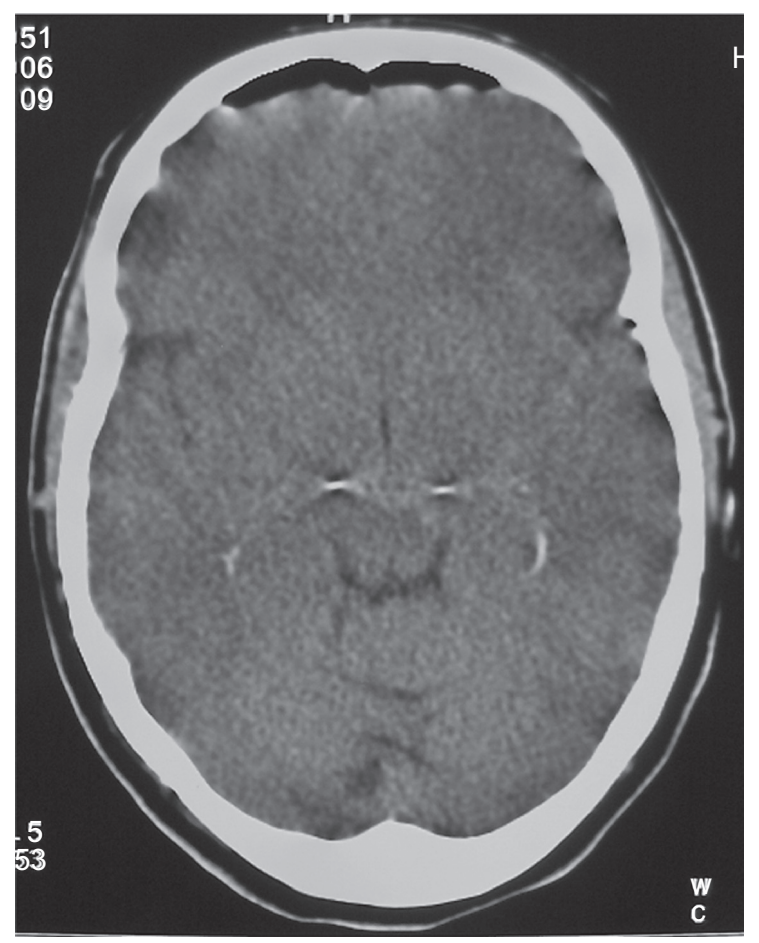

Figure I Early postoperative computed tomography scan documenting correct electrodes positioning. macrostimulation. The left side was operated last: in order to minimize cerebrospinal fluid leakage, bony chips obtained from the burr hole, together with fibrin glue and oxidized cellulose were packed together in order to seal the burr hole during electrode advancement towards the target. The central track was chosen for each side, and the definitive electrode positioning was followed and confirmed under fluoroscopy. The time required for surgery was five hours and the blood loss was negligible.

Immediate postoperative neurological status of the patient was followed at the neurosurgical ward and was negative. Four hours after surgery the patient underwent a control CT scan of the brain (Figure 1), documenting correct positioning of the stimulating electrodes. A minimal (less than $0.5 \mathrm{~cm}$ ) cortical hematoma on the left side with no mass effect was documented. One hour later, right hemiparesis and diminished level of consciousness were noticed, together with a severe dysarthria, and a further control CT scan was obtained: a $3 \mathrm{~cm}$ maximum diameter cortical-subcortical hematoma was documented, approximately $25 \mathrm{cc}$ in volume, located in the frontal lobe along the electrode's trajectory, with a significant local mass-effect (Figure 2). After careful discussion considering the absence of midline shift, and the absence of a further worsening of

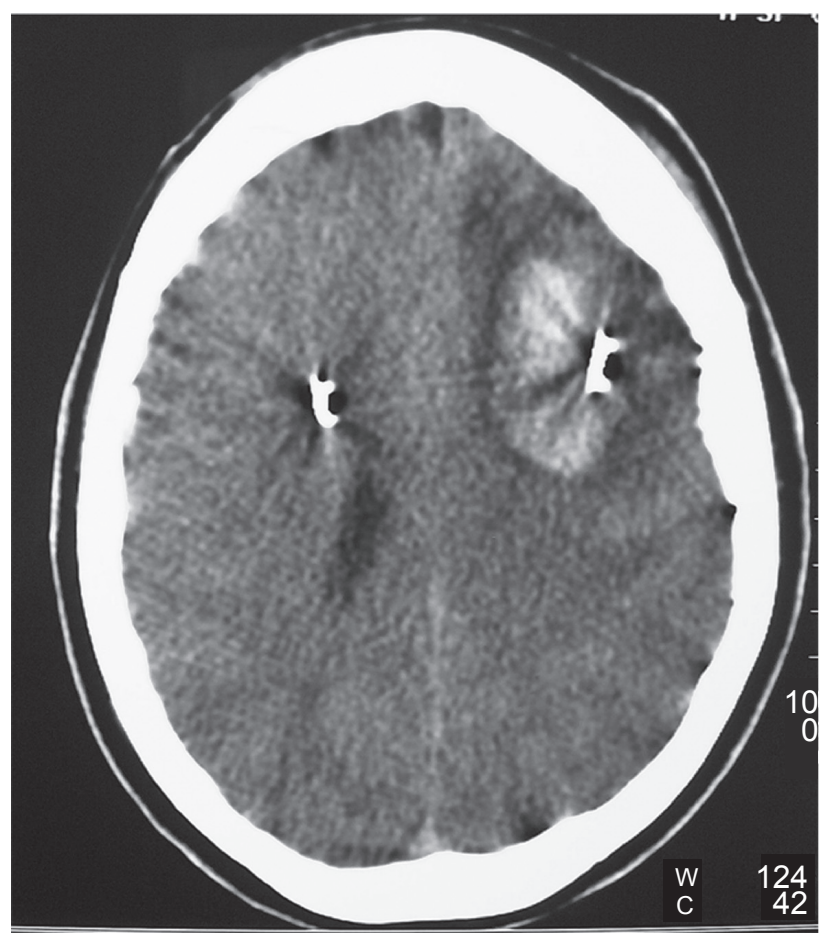

Figure 2 Computed tomography scan taken after the patient's health deteoriated, documenting frontal intraparenchymal hematoma along the trajectory of the definitive electrode. 
the clinical picture, conservative management was decided upon, and was mainly based on the stability of the clinical picture of the patient. The neurological status of the patient slowly improved over the following days, and no intensive care admission was required. At a CT scan performed eight days after STN-DBS, initial lateral displacement of the left electrode was noticed, and reached $8 \mathrm{~mm}$ lateral to the target as measured on the axial plane at a CT scan control performed during the 13th postoperative day (Figure 3). The patient was dismissed from the neurosurgical ward and sent to the Neurology Department in Istituto Mondino one month later, after a second surgical procedure for positioning of the Kinetra pulse generator (Medtronic Sofamor Danek) in a subcutaneous, subclavear pouch.

Kinetra pulse generator was activated 45 days after electrode implantation, after a control CT scan had demonstrated a complete readsorption of the hematoma. The images from this second CT scan were obtained with an inclination of the gantry which was different from the immediate postoperative CT control. To address this problem and correctly evaluate the position of the left electrode after hematoma readsorption, the images were re-calculated with the aid of dedicated software (SPM5; Wellcome Trust Centre for Neuroimaging, London, UK and Matlab 7.1; Mathworks Inc., Natick, MA, USA), applying roto-traslations to the CT voxels to obtain slices which were comparable to the pre-hematoma pictures (Figure 4). Comparison between the

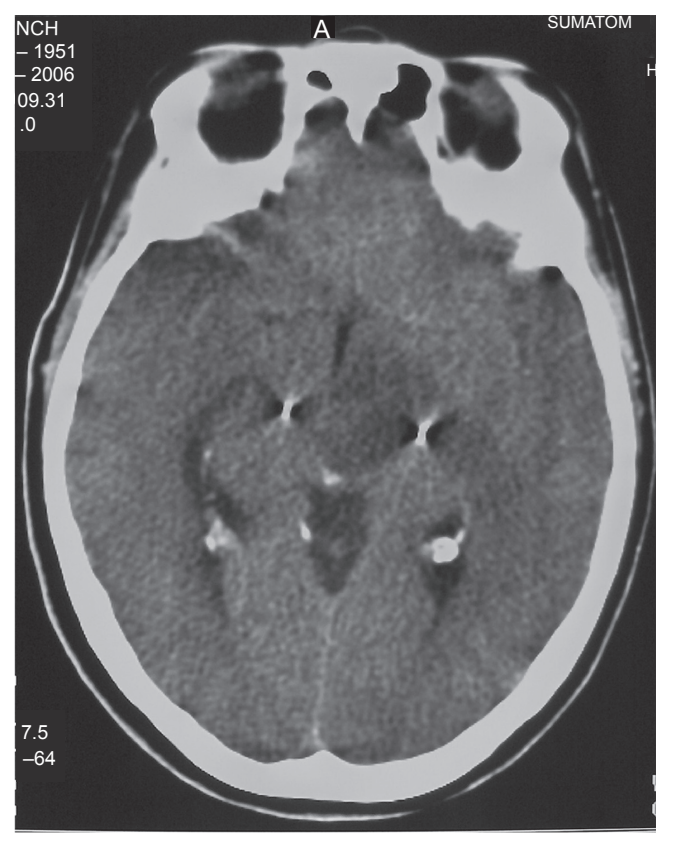

Figure 3 Maximum electrode displacement, as noted on the 13th postoperative day control computed tomography scan. two exams documented return of the electrode to a position comparable to the immediate postoperative control.

The patient underwent an intensive rehabilitation programme, with progressive improvement of the right pyramidal syndrome, that six months after the hemorrhage, consisted in a slight hypostenia at right limbs, slight dysphagia, and serious dysarthria.

At the evaluation performed six months after surgery, the UPDRS part III score was 20 in ON therapy/ON stimulation, 25 in OFF therapy/ON stimulation, 22 in $\mathrm{ON}$ therapy/OFF stimulation, and 35 in OFF therapy/OFF stimulation OFF phase. Involuntary movements were absent, such as OFF phases. The Mini Mental State Examination score was 30 (range 26-30). Clinical features were characterized by slight hypertonia and hypokinesia. The severe trunk dystonia was remitted. The antiparkinsonian therapy consisted in levodopa/benserazide 150/37.5 mg four times a day and $1 \mathrm{mg}$ of cabergoline twice a day.

Stimulation parameters were set for bilateral bipolar stimulation (2+1- and 6+5-) at $3.2 \mathrm{mV}$ of amplitude, $180 \mathrm{~Hz}$ of pulse frequency and 60 microseconds of pulse width after careful regulation, considering side effects and clinical improvement. These settings didn't give rise to side effects.

\section{Discussion}

Intraparenchymal hemorrhage is considered the most important complication related to DBS procedure, although

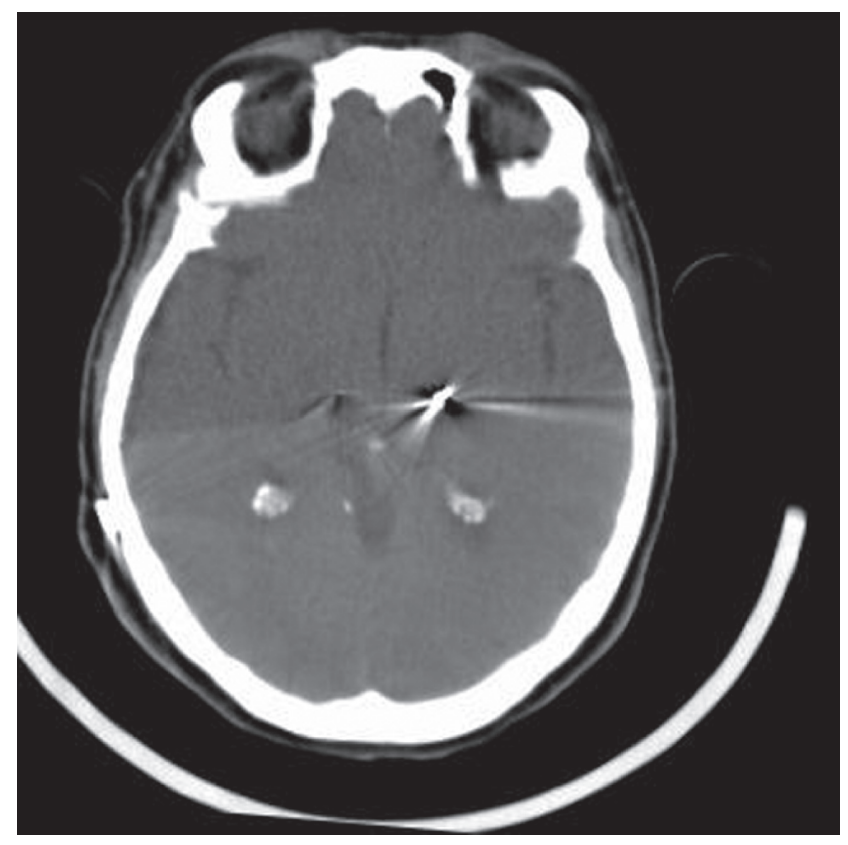

Figure 4 Forty fifth postoperative day control computed tomography scan demonstrating return of the left definitive electrode to the original position. 


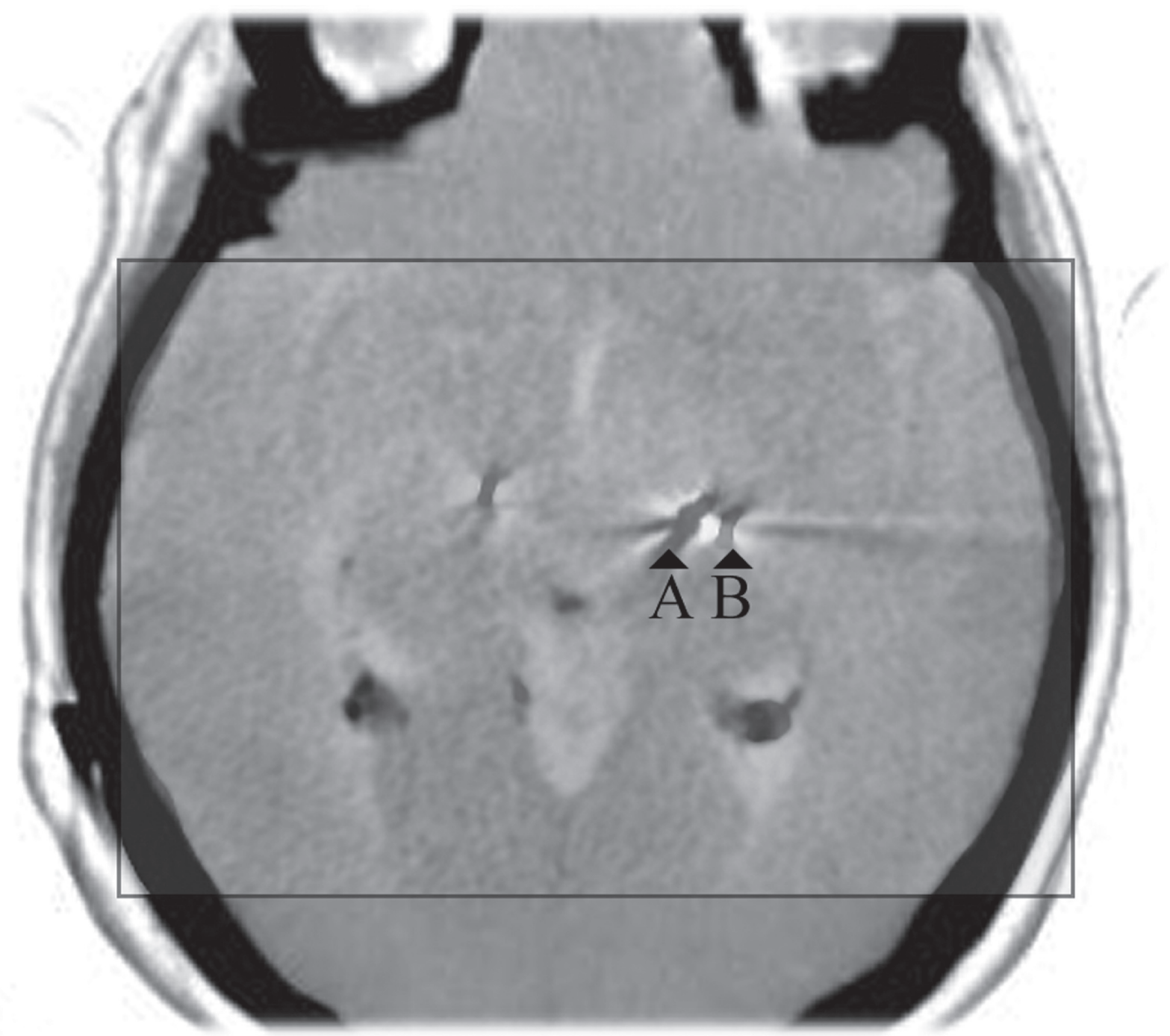

Figure 5 Negative images of the immediate postoperative control and of prolonged follow-up computed tomography (CT) scan are superimposed and scaled (in the square). " $A$ " refers to the electrode in the final position, which is symmetrical to the contralateral, while " $B$ " refers to the electrode as represented in the immediate postoperative CT control in which the hematoma had been demonstrated. Note the shift of the electrode compared to the image referred to as "A". Third ventricle, choroidal plexuses, cerebellar vermis, cranial bone, and contralateral electrode are superimposed.

it can occur without clinical worsening. ${ }^{6}$ According to Binder and colleagues ${ }^{7}$ and to other reports, ${ }^{6,8-10}$ its incidence is believed to be up to $3.3 \%$, with a $0.6 \%$ risk of developing new neurological deficits. There is a lack of data in the literature concerning electrode displacement as a consequence of such an adverse event. Our results on a large series of DBS procedures with this technique are comparable with those of well-experienced centers: ${ }^{11}$ this is the only case of electrode displacement observed in our series of 313 procedures. All these patients at our functional neurosurgery facility are screened preoperatively for coagulopathy, uncontrolled hypertension, or recent use of antiplatelet agents. Neuronavigation is performed on a gadolinium-enhanced MRI scan of the brain in order to obtain the safest trajectory for the electrodes.
Recently, multi-track microrecording exploration has been put in correlation with an increased risk of hemorrhage. ${ }^{12}$ In order to minimize mechanical stress, acquisition is performed for a minimum of two minutes every $0.5 \mathrm{~mm}$ advancement of the exploring heads. ${ }^{7}$ Therefore it is difficult to determine the exact cause of hematoma formation in this case, which is still to be traced back mainly to mechanical stress and not to infarction of an ischemic area. Evaluating late imaging results, the displacement of the stimulating electrode is probably to be considered a "false image", due to oedema of basal ganglia structures, rather than an effect of mechanical distraction exerted by intraparenchymal blood collection, and therefore not a real displacement of the electrode from the parenchymal structures. Vasogenic edema resolution obtained 
by careful conservative management of the patient eventually led the electrode to its correct positioning, thus avoiding a second surgical procedure which was, in fact, unnecessary. Moreover, in our opinion, no guidelines could be given solely taking into account features of the neuroradiological picture such as blood collection volume or electrode displacement and without considering the evolution of the clinical picture of the patient. ${ }^{13-15}$

\section{Financial disclosure}

No financial support has been received by the authors involved with the present paper. The authors declare no conflict of interests.

\section{Acknowledgments}

We would like to acknowledge Ms Olga Lee Rachello for English revision.

\section{References}

1. Beric A, Kelly PJ, Rezai A, et al. Complications of deep brain stimulation surgery. Stereotact Funct Neurosurg. 2001;77(1-4):73-78.

2. Fahn S, Elton RL; members of the UPDRS Development Committee. Unified Parkinson's Disease Rating Scale. In: Fahn S, Marsden CD, Calne DB, Lieberman A, editors. Recent Developments in Parkinson's Disease. Florhan Park, NJ: McMillan Health Care Information; 1987. p. 157-163.

3. Goetz CG, Stebbins GT, Shale HM, et al. Utility of an objective dyskinesia rating scale for Parkinson's disease: inter- and intrarater reliability assessment. Mov Disord. 1994;9:390-394.
4. Folstein MF, Folstein SE, McHugh PR. "Mini-mental state." A practical method for grading the cognitive state of patients for the clinician. J Psychiatr Res. 1975;12:189-198.

5. Coenen VA, Gielen F, Rohde I, et al. Subtalamic nucleus stimulation for advanced Parkinson's disease: how to find a far medial STN. Minim Invasive Neurosurg. 2004;47(6):373-377.

6. Goodman RR, Kim B, McClelland S 3rd, et al. Operative techniques and morbidity with subthalamic nucleus deep brain stimulation in 100 consecutive patients with advanced Parkinson's disease. J Neurol Neurosurg Psychiatry. 2006;77(1):12-17.

7. Binder DK, Rau GM, Starr PA. Risk factors for hemorrhage during microelectrode-guided deep brain stimulator implantation for movement disorders. Neurosurg. 2005;56(4):722-732; discussion 722-732.

8. Deuschl G, Volkmann J, Krack P. Editorial: Deep brain stimulation for movement disorders. Mov Disord. 2002;17(Suppl 3):S1-S5.

9. Pollak P, Fraix V, Krack P, et al. Treatment results: Parkinson's disease. Mov Disord. 2002;17(Suppl 3):S75-S83.

10. Fraix V, Houeto JL, Lagrange C, et al; SPARK Study Group. Clinical and economic results of bilateral subthalamic nucleus stimulation in Parkinson's disease. J Neurol Neurosurg Psychiatry. 2006;77(4):443-449.

11. Lang AE, Lozano AM, Montgomery E, Duff J, Tasker R, Hutchinson W. Posteroventral medial pallidotomy in advanced Parkinson's disease. N Engl J Med. 1997;337:1036-1042.

12. Hariz MI. Complications of deep brain stimulation surgery. Mov Disord 2002;17(Suppl 3):S162-S166.

13. Binder DK, Rau G, Starr PA. Hemorrhagic complications of microelectrode-guided deep brain stimulation. Stereotact Funct Neurosurg. 2003;80(1-4):28-31

14. Kawakami N, Jessen H, Bordini B, Gallagher C, Klootwyk J, Garell CP. Deep brain stimulation of the subthalamic nucleus in Parkinson's disease. WMJ. 2005;104(6):35-38.

15. Voges J, Waerzeggers Y, Maarouf M, et al. Deep-brain stimulation: long-term analysis of complications caused by hardware and surgery - experiences from a single centre. J Neurol Neurosurg Psychiatry. 2006;77(7):868-872. 
